\title{
Critical Conductor Temperatures in Submarine Cables Equipped with Protection Pipes
}

\author{
Rógvi Østerø, Joachim Holbøll \\ Technical University of Denmark \\ Centre for electric power and energy \\ s083436@student.dtu.dk, \\ jh@electro.dtu.dk
}

\author{
Rasmus Olsen \\ Energinet.dk \\ Denmark
}

\author{
Ivan Arana Aristi \\ Dong Energy \\ Denmark
}

\begin{abstract}
Submarine cables used in harsh environmental conditions are often strengthened by means of protective structures, in addition to the armour, for enhanced mechanical durability. The added exterior protection shields the cable against damage during installation and operation. These, polymeric or metallic protective structures, contribute to the temperature increase in the cables by reducing the natural cooling processes.

In this paper are described investigations on the thermal conditions in and outside a three-phased armoured submarine cable equipped with a fully enclosed mechanical protection. The investigations are conducted through a full-scale experiment, including mitigation techniques.

In the full-scale experiment, the effects of the protective pipe result in a temperature increase when compared to an experiment using a cable with no protective pipe. The measurements show that the addition of the mechanical protection may result in the phase conductor temperatures to reach and exceed the critical $90{ }^{\circ} \mathrm{C}$ limit, even under otherwise normal operating conditions.

The effect of measures to mitigate the increase in phase conductor temperature was also investigated. At set intervals a symmetrical distribution of holes were drilled into the protective pipe. This mitigation technique resulted in a temperature decrease of approximately $9{ }^{\circ} \mathrm{C}$ in phase conductor temperature.
\end{abstract}

\section{Introduction}

The rapid increase in installed offshore wind farms around the globe calls for a greater optimization of all components. The endeavour to optimise components in a wind farm often presents issues in how components influence each other. At the offshore windfarm at Anholt in Jutland, Denmark, the submarine transmission cables utilised were covered with a mechanical protect, also known as J-tubes, the J-tube covered the cable from the seabed and into the wind turbine monopole. This is a commonly utilised method to protect the cable against damage during installation and operation. The J-tube was fixed onto the hang-off and therefore formed a complete airtight envelopment around the cable. It was estimated that the fully enveloping J-tube had a negative effect on the current carrying capacity of the cable. There was the assumption, that the added protection added to the thermal insulation of the cable. Added insulation would mean increased temperatures and therefore limiting the current carrying capacity of the cable. It is typically recommended, by cable manufacturers, that the core temperature of a crosslinked polyethylene cable should not exceed $90{ }^{\circ} \mathrm{C}$. This recommendation links to the aging of the cross-linked polyethylene, which accelerates at higher temperatures [1]. Diminution of the current carrying capacity, due to thermal limitations, could ultimately necessitate an increase of conductor cross-section, meaning increased costs. Therefore it was deemed prudent to determine the extent of the thermal effects by the added protection, and to propose a method of mitigating the effects. This presents the aim of this paper, which is to present the impact a J-tube has on the core temperature in a submarine cable through a full-scale experiment. The experiment covered in this paper, concerned with the vertical entry of three-phased cables in J-tubes. This experiment was a part of a larger experiment see Fig. 1. The larger experiment covered three scenarios, which a submarine cable undergoes in an offshore wind turbine. The first scenario involved the cable covered with a polymer pipe submerged in a water-filled sandbox. This was to simulate a mechanically protected cable dug down in the seabed.

The second scenario involved a caterpillar type like mechanical protection in a box filled with water. This simulated the cables transition from seabed to sea.

The third scenario involved the submarine cable suspended in mid-air covered with a polymer pipe. This scenario simulated the protected cable within the wind turbine monopole. 


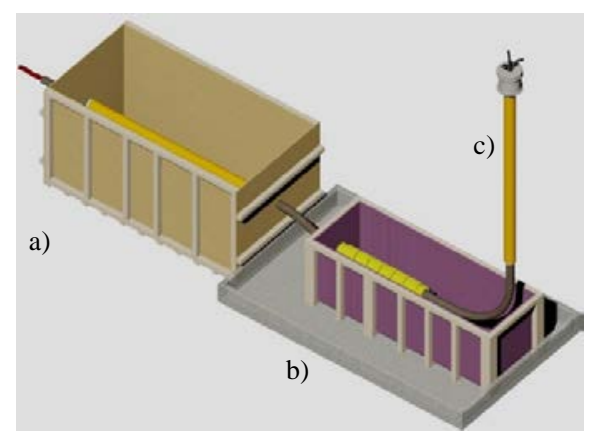

Figure 1 - Illustration of the entire full-scale experiment. a) The sandbox. b) The water box. c) The vertical entry of the three-phased cable in $J$-tubes.

\section{Experiment Setup}

As stated, the experiment was to determine the thermal effects of introducing mitigating initiatives into the cable's mechanical protection system. It was therefore decided to perform temperature measurements, of a heavily loaded cable, inside the polymeric pipe. The temperature measurements were performed in two configurations:

1. Unimproved cooling - standard installation

2. With improved cooling - including mitigating initiative.

The details of the experimental setup are given in the following.

\section{Component data}

The experimental setup consisted of a three-phased armoured submarine cable covered by a polymer pipe.

The cable specifications:

- Three phase

- Conductor cross-section $500 \mathrm{~mm}^{2}$

- $\quad$ Stranded

- $20 / 34 \mathrm{kV}$

The polymer pipe specifications:

- Inner diameter $183 \mathrm{~mm}$

- Outer diameter $245 \mathrm{~mm}$

- Wall thickness $30 \mathrm{~mm}$

- $\quad$ Pipe length $3960 \mathrm{~mm}$

\section{Temperature measurements}

Facilitating the temperature measurements are thermocouples of the type $\mathrm{K}$ and the logger Hioki LR8400-20 collects and logs the measurement data.

The areas fitted with thermocouples are the respective top and bottom areas of the covered cable, see Fig. 2 .

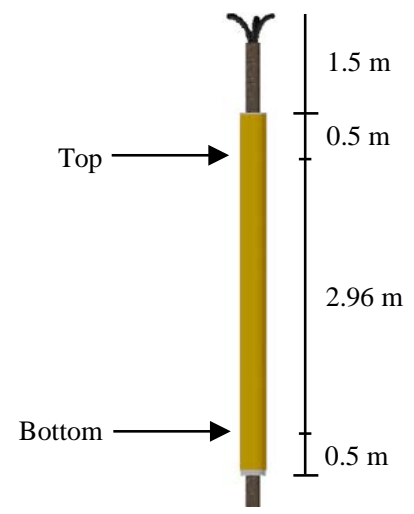

Figure 2 - The position of thermocouples inserted into the cable.

The thermocouples measured the temperatures for the following areas/layers:

- Submarine cable
o Conductors
o Sheath
o Armour
o Fibre optics tube
o Surface

- Ambient

To accommodate the measurements of the temperature of the cable conductor, sheath and armour, thermocouples were inserted into the cable layers. This was done by locating the individual phases, through observations of the contour of the cable, thereafter drilling $3 \mathrm{~mm}$ holes into the cable. The holes for the respective layers were spaced $2 \mathrm{~cm}$, cable lengthwise, from each other. The holes for the conductor were drilled until the hard surface of the conductor was reached. The holes for the sheath were drilled with the sheath and the drill connected through a multimeter set to a continuity measurement. This was to verify if the sheath was reached. For the armour, a hole was drilled between the armour wires. The sensors were inserted into the holes, see Fig. 3, and the hole was thereafter filled with a polymer compound.

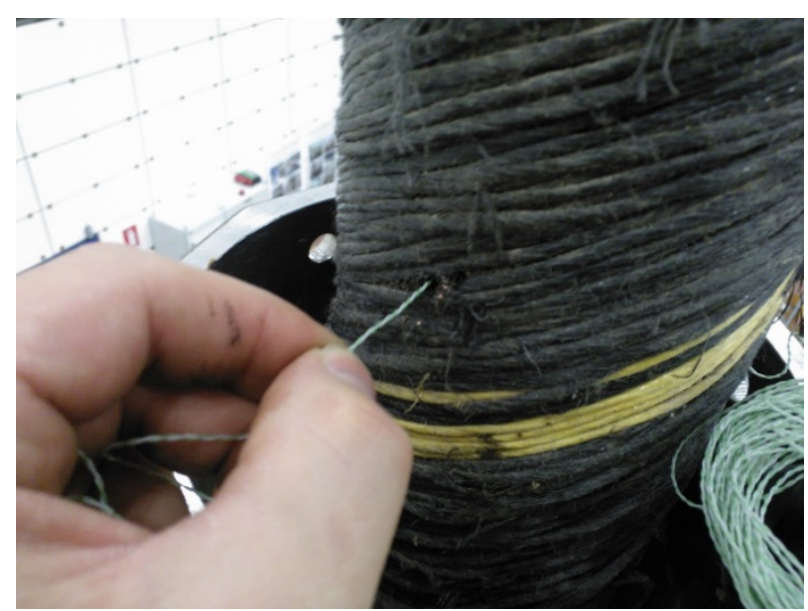

Figure 3 - The insertion of the thermocouple into the cable.

The surface sensors were fixed to the surface of the cable by means of duct tape. The insertion of the 
temperature sensor into the fiberoptics tube involved the removal of the optical fibres and thereafter the insertion of the sensor.

With the thermocouples inserted into their respective areas the polymer pipe ends were sealed with a polyurethane foam compound, fully enclosing the polymer pipe around the cable, see Fig. 4.

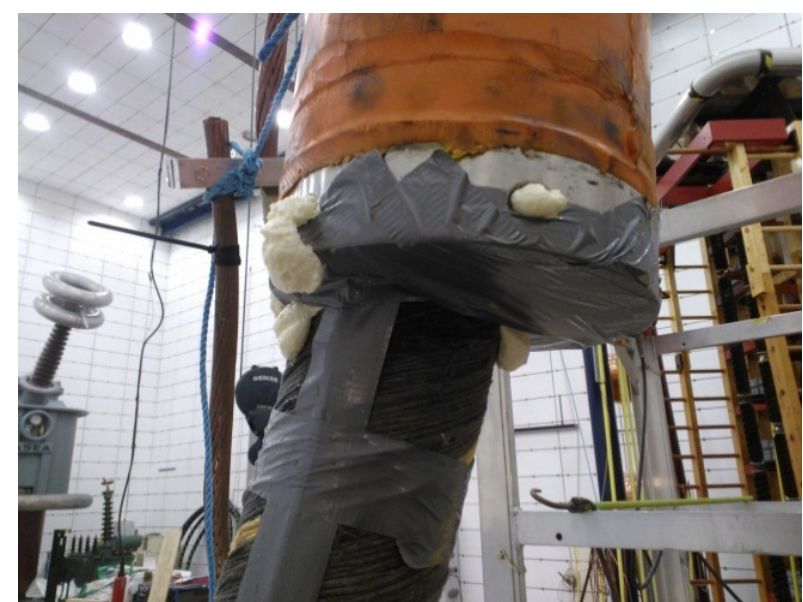

Figure 4 - Picture of the bottom end of the polymer pipe fully enclosed around the cable.

\section{Electrical circuit}

At the source end, the three cable conductor phases are each extended with two $150 \mathrm{~mm} 2$ flexible copper conductors. Here the flexible copper conductors are lead through current transformers, one transformer for each phase conductor. Hereafter the phases are star connected by means of a large aluminium clamp, see Fig. 5 for a picture of the electrical setup and Fig. 6 for a circuit diagram of the setup.

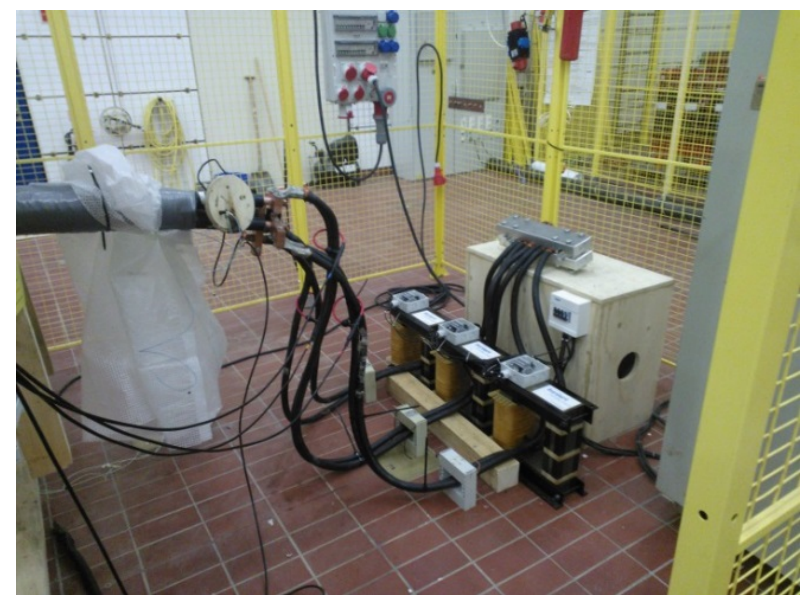

Figure 5 - Picture of the electrical setup at the source end

The conductors at the top of the experimental setup are star connected by means of copper clamps. The cable sheaths are solidly bonded. An illustration of the electrical circuit is displayed in Fig. 6.

$$
\text { Cable }
$$

Current transf.

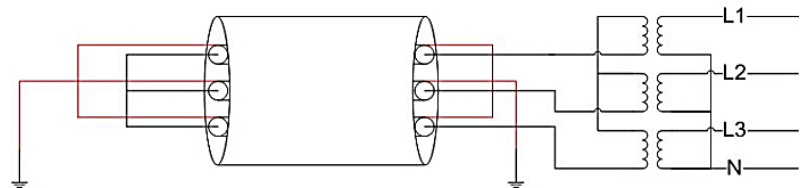

Figure 6 - Electrical circuit diagram of experimental setup

The three current transformers where connected to a variable three-phased transformer, enabling the control of the three individual current transformers.

\section{Current measurements}

To measure the current during the experiments two methods of measuring the current where utilised. During the experiment, without improved cooling, Rogowski coils where utilised. For the second experiment with improved cooling current coils were utilised. This was due to that another project required the Rogowski coils and therefore mandated a second method. In the unimproved cooling experiment the Rogowski coils where connected to an Agilent DSO-X 3024 oscilloscope. The oscilloscope connected to a computer, where a Labview program was set to log the current data. The Rogowski coils measured the conductor and the sheath current, see measurement configuration in Fig. 7.

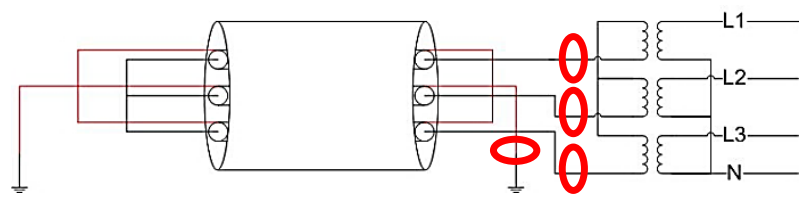

Figure 7 - Illustration of the position (red circles) for the current measurements.

For the improved cooling experiments, current coils were utilised and thereafter the resulting current was measured by Agilent U1212A multimeters, which also logged the current data. During both experiments, the currents were double-checked with the same handheld clamp amp meter, ensuring correspondence.

\section{Experiment procedure}

With the experimental setup, two different thermal configuration were investigated.

Firstly, one without improved cooling, where the pipe was installed as normally done in wind turbines.

Secondly, one with improved cooling, where at set intervals, a symmetrical distribution of holes were drilled into the protective pipe.

\section{Unimproved cooling experiment}

This experiment was initiated by starting the temperature logging and waiting for the setup to obtain a steady state temperature equal to the ambient temperature. After a few hours of logging the temperatures a current of $684 \mathrm{~A}$ is applied to the conductors. The current of 684 A was set by Dong Energy, due to the load characteristics of the cable. The experiment was set to run until the conductors reached a steady state temperature, and thereafter kept for a duration of 7 days. 
The procedure for the unimproved cooling experiment:

- $\quad$ Start measurements

- Ambient steady state

- Applying a current of $684 \mathrm{~A}$

- Steady state

- $\quad$ Stop experiment

\section{With improved cooling}

This experiment was to some extent conducted in the same manner as the previous, but with the slight change of introduced holes into the polymer pipe. The holes would create natural convection within the pipe. The improved cooling is applied by drilling $20 \mathrm{~mm}$ holes into the polymer pipe. This experiment was initialised by firstly drilling three holes in each end of the polymer pipe. Thereafter, the temperature was logged and when the setup had reached a steady state temperature the current of 684 A was applied. In the course of every approx. 24 hours, additional holes were introduced into the polymer pipe. The configuration and distribution of the holes are viewed in Fig. 8.

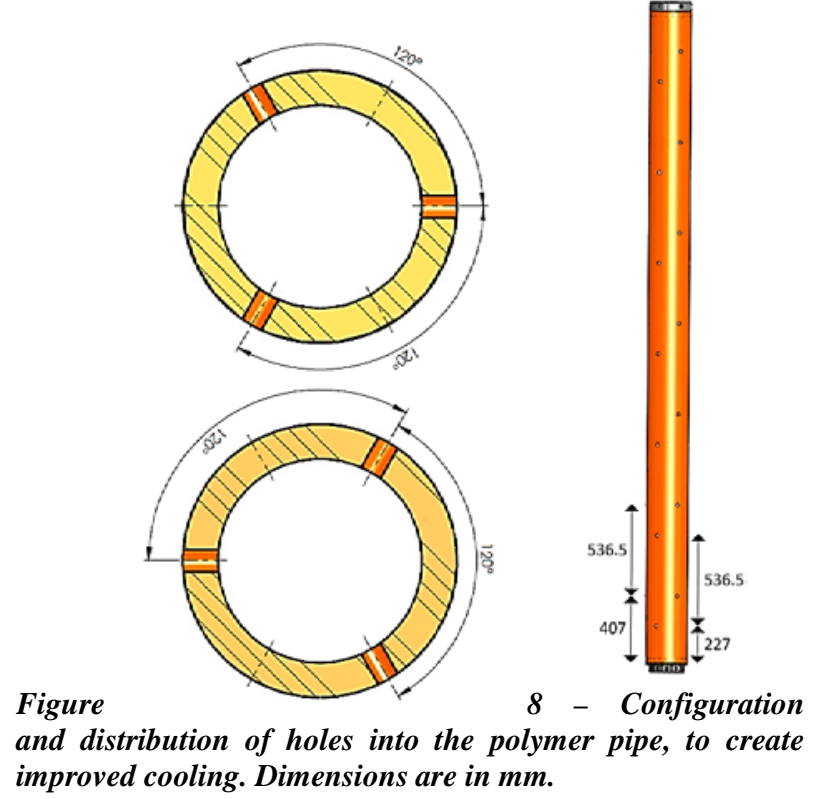

The procedure for the improved cooling experiment:

- First set of holes introduced into the polymer pipe

- Ambient temperature steady state

- Applying a current of $684 \mathrm{~A}$

- Drilling three $20 \mathrm{~mm}$, in each end of the polymer pipe every $\sim 24 \mathrm{~h}$.

- Steady state

- $\quad$ Stop experiment

\section{Measurement results}

The results of the measured temperatures, at the top and bottom of the cable, for both the unimproved cooling experiment and the improved cooling experiment, are shown in the following. The plots included in this section, include the maximum conductor temperature, ambient temperature, maximum conductor temperature with the ambient subtracted and the applied current.

\section{Unimproved cooling experiment results}

Here the results are plotted and depicted as Fig. 9 and Fig. 10. Fig. 9 displays the measured temperatures and currents for the top area and Fig. 10 for the bottom area.

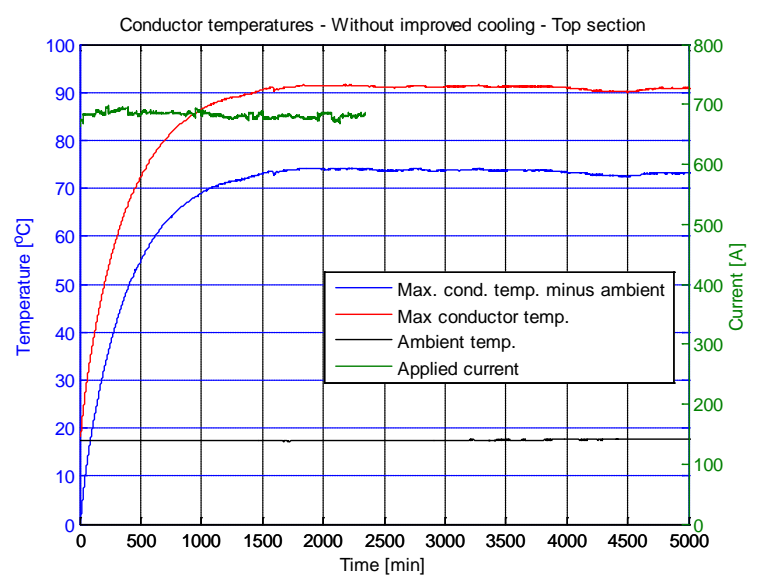

Figure 9 - Temperatures and the current for the top area of the unimproved cooling experiment.

By observing Fig. 9 it is apparent that with the applied current of 684A, the temperatures reaches a steady state temperature after a duration of approximately $2000 \mathrm{~min}$. The red line depicts the maximum conductor temperature and here the temperature exceed the critical limit of $90{ }^{\circ} \mathrm{C}$. This shows that there are some consideration to be made about the conductor dimensions. The blue line depicts the conductor temperature with the ambient temperature subtracted. Here it is observed that the maximum temperature is at around $73{ }^{\circ} \mathrm{C}$. It is also noticeable that the current measurements stop at around $2300 \mathrm{~min}$. This is due to technical issues. Even though up to this point the conductor temperature is stabilised and the current does not show any significant changes.

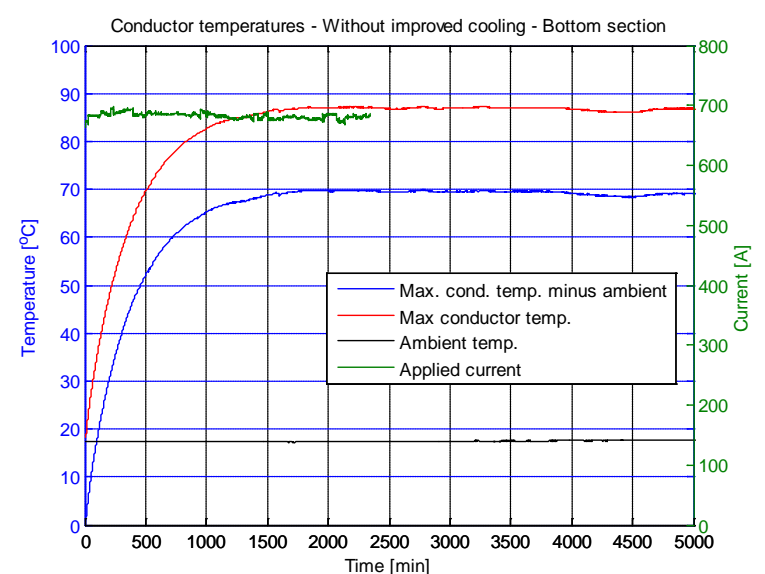

Figure 10 - Temperatures and the current for the bottom area of the unimproved cooling experiment.

In Fig. 10, it is observed that the conductor temperatures for the bottom area show lower temperatures compared to the top section. Here the maximum conductor temperature is approximately $86{ }^{\circ} \mathrm{C}$. With the ambient temperature subtracted, the conductor temperature is around $70{ }^{\circ} \mathrm{C}$. 
Improved cooling experiment results

Here the results are plotted and depicted as Fig. 11 and Fig.12. Fig. 11 displays the measured temperatures and currents for the top area and Fig. 12 the bottom area.

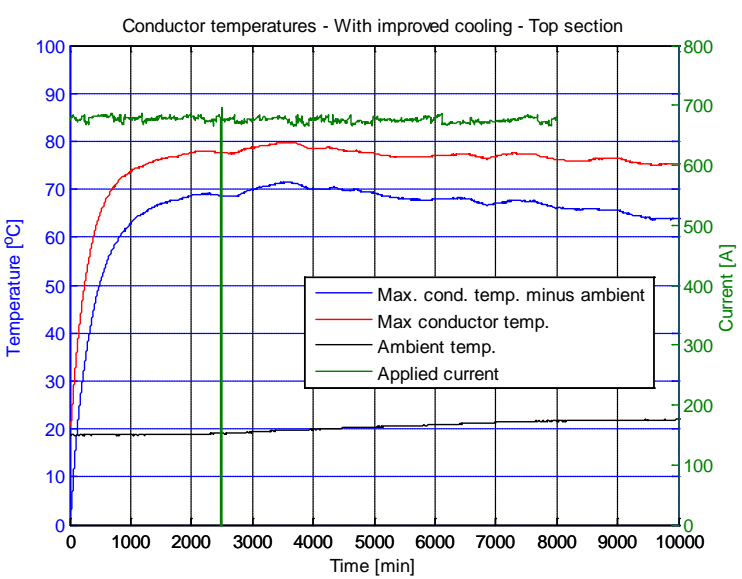

Figure 11 - Temperatures and the current for the top area of the improved cooling experiment.

Observing Fig. 11, here the conductor temperatures reach a steady state at around $2000 \mathrm{~min}$. It is also apparent that between 0 and $2000 \mathrm{~min}$ the conductor temperature is comparably lower than in the previous experiment. This shows that by just drilling three holes in each end leads to a decrease in temperature. Fig. 11 also shows that by introducing more and more holes into the polymer pipe there is a continuous decrement in temperature. The final holes are introduced at the around 9000 min marker. After 9500 min the conductor temperature reaches a steady state with a maximum temperature of around $64^{\circ} \mathrm{C}$. Compared to the previous experiment this is a temperature decrease of around $9^{\circ}$ C.

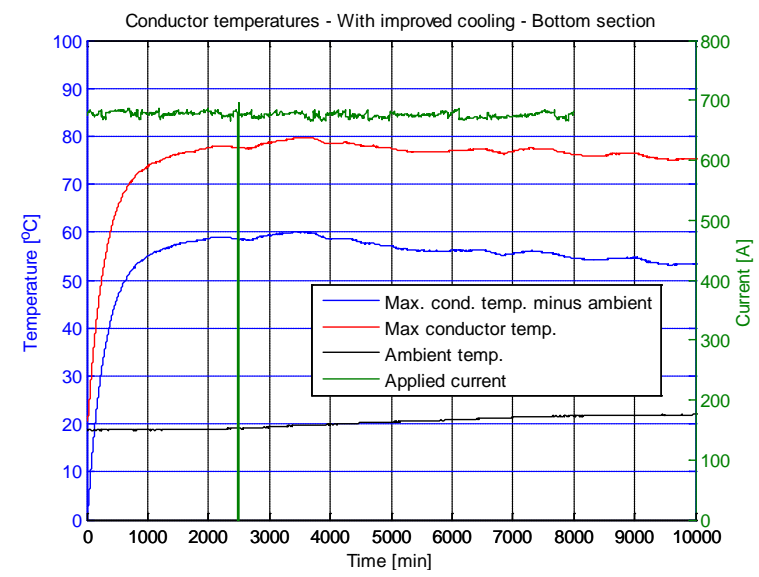

Figure 12 - Graph depicts the temperatures and the current for the bottom area of the improved cooling experiment.

Observing Fig. 10, it is apparent that between 0 and 2000 min the conductor temperature is comparably lower than in the previous experiment. This figure also shows lower temperatures compared to the first experiment.

\section{Discussion}

\section{Ambient temperature}

The ambient temperatures differed in the two experiments. The experiments were conducted in early spring and late spring. The ambient temperature varied by approximately $3{ }^{\circ} \mathrm{C}$ from the start of the first experiment to the end of the last experiment. Although this was not considered to influence the experiments that much.

\section{Current measurements}

Considering the current measurements it is noted, that there are two methods of measuring the current. Moreover, due to technical malfunctions the current measurements do not cover the entire duration of the experiments. The inability of surveying the current is unfortunate, especially during the improved cooling experiment. Here, the conductor temperature has not fully reached a steady state, when the current measurements fail. Although the current measurements only cover the initial period of the experiment, it is stressed that the current is steady throughout the measurement range. During this time, the conductor temperature continuously decreases and reaches a steady state shortly after the current measurements fail. Therefore, it is considered that the current measurements depict the important period of the experiment.

\section{Mitigating effect of temperature increase}

The results clearly indicate that there is a decrease in conductor temperature during the mitigation trial. This reduction in conductor temperature would have positive effects. Firstly, a decrease in temperature would enable the cable to carry a larger current. But also, with the improved thermal conditions of the J-tube, the cables conductor cross-section could be reduced. A reduction in cross-section means less material is utilised to makeup the cable. This would be a significant economic advantage, reducing cost in the submarine cable department. Any economic advantage is to be embraced, since they could make or break a project.

\section{Conclusion}

This paper has through full-scale experimental studies, shown that the temperature of three-phased power cables, in the top of the J-tube of a wind turbine, can be reduced. This could lead to economic advantages and possibly decrease costs for offshore wind farms. The experiment without improved cooling ascertained that, the top of the polymer pipe showed the highest temperatures. Here it was also concluded, that the conductor temperature at the top exceeded the critical limit of $90^{\circ} \mathrm{C}$, recommended by cable manufactures.

The experiment with improved cooling showed that by introducing holes into the polymer pipe, the conductor temperature decreases. More specifically the conductor temperatures are lowered by around $9{ }^{\circ} \mathrm{C}$. This goes to show that adding a fully enclosed polymer pipe onto a submarine cable, does in fact increase the conductor 
temperature during operation. Moreover, by breaking the enclosure through the introduction of holes, the $\mathrm{J}$ -

Tubes limiting abilities are reduced, enabling a possible economic advantage.

\section{References}

[1] Olsen, R. S., Holboell, J., Henriksen, M., Hansen, Z. J. Thermal Ageing of XLPE Cable Insulation under Operational Temperatures -Does It Exist?, Nordic Insulation Symposium 2013 